\title{
Breaking down brain barrier breaches in cerebral malaria
}

\author{
Jens E.V. Petersen, ${ }^{1}$ Thomas Lavstsen, ${ }^{1}$ and Alister Craig ${ }^{2}$ \\ 'Centre for Medical Parasitology, Department of Immunology and Microbiology, Faculty of Health and Medical Sciences, University of Copenhagen, and \\ Department of Infectious Diseases, Rigshospitalet, Copenhagen, Denmark. 'Liverpool School of Tropical Medicine, Liverpool, United Kingdom.
}

Recent findings have linked brain swelling to death in cerebral malaria (CM). These observations have prompted a number of investigations into the mechanisms of this pathology with the goal of identifying potential therapeutic targets. In this issue of the $J C l$, Gallego-Delgado and colleagues present evidence that implicates angiotensin receptors and the relocation of $\beta$-catenin to the endothelial cell nucleus in $\mathrm{CM}$. This study provides a renewed focus on infected erythrocyte debris as the cause of endothelial damage and challenges previous work implicating direct effects of infected erythrocyte sequestration in the brain as the major driver of disease. While this work provides potential therapeutic avenues for $\mathrm{CM}$, it leaves a number of questions unanswered.

\section{Cerebral malaria}

The management of patients with severe infections of the malaria parasite Plasmodium falciparum remains a major challenge in the combat against malaria-related death. Despite the ability of current antimalarial therapies to clear the infecting parasites, patients who receive treatment after the onset of severe manifestations still have a high mortality rate. For example, patients with cerebral malaria $(\mathrm{CM})$, which is characterized by impaired consciousness, have an $18 \%$ mortality rate, even when given artemisinin-based combination treatments (1). At present, no targeted adjunctive therapies, aside from supportive care and fluid management, are available for those that present with this complication.

$\mathrm{CM}$ is associated with disruption of the blood-brain barrier (BBB), various degrees of hemorrhagic lesions, localized vascular thrombosis, and accumulation of monocytes (2). The development of CM is thought to be driven by the sequestration of infected erythrocytes in the brain, although the relative importance of the sequestration itself and an unfavorable inflammatory immune response in the brain as a result of sequestration remains a much debated topic. Recently, work looking at associations with severe malaria has suggested that microvascular obstruction and endothelial activation are independent variables in CM pathology (3).

MRI scans of children who died from $\mathrm{CM}$ suggest that respiratory arrest due to brain swelling is a major cause of death (4). So, although the complete picture of pathogenesis remains to be elucidated, and the disruption of the BBB or even edema is unlikely to be the only cause of $\mathrm{CM}$, strategies aimed at maintenance of $\mathrm{BBB}$ integrity might be sufficient to offset some of the pathophysiological changes caused by $P$. falciparum infection, including brain edema and even death.

In regard to preserving $\mathrm{BBB}$ function, the renin-angiotensin system (RAS) may be an interesting target for adjunctive therapies for $\mathrm{CM}$, as angiotensin receptor blockers (ARBs) have been shown to protect the barrier integrity of brain endothelial cells in vitro (5). While ARBs inhibit angiotensin II-mediated barrier

Related Article: p. 4016

Conflict of interest: The authors have declared that no conflict of interest exists.

Reference information: / Clin Invest. 2016;126(10):3725-3727. doi:10.1172/JCI90188.

disruption, these blockers will need to inhibit the disruption of the endothelial barrier caused by malaria infection to be feasible for treatment of CM.

\section{Parasite-infected erythrocyte rupture and CM development}

In this issue, Gallego-Delgado and colleagues have demonstrated that the rupture of malaria-infected erythrocytes causes endothelial cell apoptosis and have linked these events to the translocation of $\beta$-catenin from the intercellular junctions to the cell nucleus, where it functions as a transcription factor (6). Moreover, the signaling cascade that results from the translocation of $\beta$-catenin to the nucleus and the subsequent death of endothelial cells could be interrupted by blockade of the angiotensin II type 1 receptor (AT1) or stimulation of the angiotensin II type 2 receptor (AT2), both of which are present on the surface of these cells.

In line with earlier studies $(7,8)$, Gallego-Delgado et al. also determined that $\mathrm{BBB}$ disruption requires erythrocyte rupture, as adhesion of trophozoite-stageinfected erythrocytes, and erythrocytes infected with parasites arrested at the schizont stage, did not result in the loss of barrier integrity or translocation of $\beta$-catenin to the nucleus (6). While Gallego-Delgado and colleagues argue that sequestration of infected erythrocytes in the brain is likely required to allow the soluble factors released by rupturing infected erythrocytes to reach damaging concentrations, their findings contradict other studies that have shown that the endothelial barrier can be disrupted without the rupture of infected erythrocytes. In vitro data suggest that the $P$. falciparum adhesion protein PfEMP1 increases susceptibility to thrombin-induced barrier disruption $(9,10)$ by blocking activated protein $\mathrm{C}$ (APC) functions, and thus the $\mathrm{BBB}$ can be indirectly affected by the adherence of infected erythrocytes. This susceptibility is under- 
lined by the loss of available endothelial protein $\mathrm{C}$ receptor (EPCR) on the surface of vessels in the brain, which occurs in conjunction with the sequestering of infected erythrocytes in vivo (11). The added insult of soluble factors that results from bursting infected erythrocytes, as reported by Gallego-Delgado et al. (6), could be augmented by the adherence of infected erythrocytes to endothelial cells, resulting in accumulating endothelial activation, barrier disruption, vascular damage, and subsequent CM. Furthermore, APC has been shown to inhibit the ability of released plasmodial and human histones to activate the endothelium (8), and thus impairing protein $\mathrm{C}$ activation might exacerbate the effect of histones, which are a candidate for the factor that is locally released by erythrocyte rupture and leads to CM-associated damage in the brain.

Data presented by Gallego-Delgado et al. suggest that only endothelial cells that originate from the brain are affected by rupturing erythrocytes (6). It will be very interesting if this hypothesis is correct; however, technical issues relating to the use of primary, nonbrain cells and immortalized brain endothelial cells provide an alternative explanation for these results. The inconsistent association between overall parasitemia and CM observed in patients (12) prompts the question of whether the rupture of infected erythrocytes alone entirely explains the loss of brain endothelial barrier integrity in CM. A simple answer for the observed tissue-specific response in $\mathrm{CM}$ is that edema in the brain, unlike in other organs, can be particularly detrimental because of the confines of the skull and thus brain endothelial cells have specific responses to stimuli. This interpretation would still not explain the difference in $\mathrm{CM}$ development and clinical outcome seen in patients with equivalent parasitemia, unless the nature of soluble factors differs between parasite isolates or, perhaps more likely, there are differences in the host response to these factors. Further studies of the localization of $\beta$-catenin in endothelial cells from postmortem CM cases could help illuminate the endothelial responses in these patients, including those with little or no vascular pathology. Moreover, such examinations of postmortem tissue may further clarify the feasibility of targeting the RAS system for treating CM.

\section{AT1 and AT2 as potential therapeutic targets}

Gallego-Delgado and colleagues provide further evidence to substantiate the potential utility of targeting the AT1 and AT2 pathway as an adjunctive therapy strategy for CM. Specifically, they showed a marked reduction of $\mathrm{CM}$-associated mortality in C57BL/6J mice infected with Plasmodium berghei ANKA in response to administration of the AT1 blocker irbesartan or the AT2 agonist compound 21, along with chloroquine, compared with infected animals treated with chloroquine alone (6). Regardless of the discrepancies in etiology of mouse experimental model and human $\mathrm{CM}$, these results are encouraging, as they suggest a potentially safe option for adjunctive therapies that may have a quicker path toward clinical application through drug repurposing. Moreover, these results add to the collection of therapeutic avenues based on targeting of the EPCR/PAR axis $(13,14)$.

Indeed, evidence of an overlap between the AT1/AT2 and EPCR pathways has been presented and has implications for malaria. Angiotensin II has been shown to upregulate angiopoietin 2 in bovine retinal endothelial cells through AT1 (15). Angiopoietin 2 is released upon endothelial activation, for example through PAR1 cleavage by thrombin, and is closely associated with the presentation of severe malaria symptoms, including CM. Moreover, aside from reducing the inflammatory effect of histones released from rupturing erythrocytes, APC also signals through sphingosine-1-phosphate receptor 1 (S1P1) to stabilize the endothelial barrier (16). In recent studies, artemisinin has been shown to downregulate $\beta$-catenin (17) and protect the BBB after a subarachnoid hemorrhage in rats. APC-mediated protection involved the S1P1/phosphatidylserine pathway and subsequent stabilization of $\beta$-catenin at intercellular junctions (18). In some endothelial cells, barrier integrity is also affected by EPCR-mediated signaling through the S1P1 pathway $(16,19)$. It would thus be interesting to investigate the BBB-protective effects of APC, AT1 blockage, or AT2 agonists in combination with an artemisinin derivative, such as artesunate, compared with the quinine based treatment regimen employed by Delgado et al. (6). Such a comparison would allow investiga- tion of the hypothesis that the improved effects of artesunate over quinine (reduced mortality, faster coma recovery, and fewer neurological sequelae; ref. 1) relate, at least in part, to its effects on S1P1.

The complex pathogenesis of CM and the difficulties of examining both adhesion and inflammation in a model system make the development of novel adjunctive therapies a big challenge. However, targeting the BBB to reduce the brain swelling is a compelling strategy for this potentially deadly complication. A similar, albeit controversial, rationale for using statins and ARBs to protect the endothelial barrier from damage has been proposed for infectious diseases other than $P$. falciparum malaria, including bacterial sepsis and Ebola (20). While each infectious disease provides specific challenges for proving the feasibility of BBB-protective therapies, the development of better strategies to circumvent breaches of the BBB is certainly attractive for all.

\section{Acknowledgments}

TL and JEVP receive research funding support from the Lundbeck Foundation, Danish Medical Research Council, and NIH. AC receives research funding support from the Wellcome Trust.

Address correspondence to: Thomas Lavstsen, Centre for Medical Parasitology, Bartholinsgade 2, 1356 Copenhagen K, Denmark. Phone: 0045.35327677; E-mail: thomasl@sund.ku.dk.

1. Dondorp AM, et al. Artesunate versus quinine in the treatment of severe falciparum malaria in African children (AQUAMAT): an open-label, randomised trial. Lancet. 2010;376(9753):1647-1657.

2. Dorovini-Zis K, et al. The neuropathology of fatal cerebral malaria in Malawian children. Am J Pathol. 2011;178(5):2146-2158.

3. Hanson J, et al. Microvascular obstruction and endothelial activation are independently associated with the clinical manifestations of severe falciparum malaria in adults: an observational study. BMC Med. 2015;13:122.

4. Seydel KB, et al. Brain swelling and death in children with cerebral malaria. N Engl JMed. 2015;372(12):1126-1137.

5. Fleegal-DeMotta MA, Doghu S, Banks WA. Angiotensin II modulates BBB permeability via activation of the AT(1) receptor in brain endothelial cells. J Cereb Blood Flow Metab. 2009;29(3):640-647.

6. Gallego-Delgado J, et al. Angiotensin receptors and $\beta$-catenin regulate brain endo- 
thelial integrity in malaria. J Clin Invest. 2016;126(10):4016-4029.

7. Tripathi AK, Sullivan DJ, Stins MF. Plasmodium falciparum-infected erythrocytes decrease the integrity of human blood-brain barrier endothelial cell monolayers. J Infect Dis. 2007;195(7):942-950.

8. Gillrie MR, et al. Plasmodium falciparum histones induce endothelial proinflammatory response and barrier dysfunction. Am J Pathol. 2012;180(3):1028-1039.

9. Petersen JE, et al. Protein C system defects inflicted by the malaria parasite protein PfEMP1 can be overcome by a soluble EPCR variant. Thromb Haemost. 2015;114(5):1038-1048.

10. Gillrie MR, et al. Diverse functional outcomes of Plasmodium falciparum ligation of EPCR: potential implications for malarial pathogenesis. Cell Microbiol. 2015;17(12):1883-1899.

11. Moxon CA, et al. Loss of endothelial protein C receptors links coagulation and inflammation to parasite sequestration in cerebral malaria in African children. Blood. 2013;122(5):842-851.

12. von Seidlein $\mathrm{L}$, et al. Predicting the clinical outcome of severe falciparum malaria in African children: findings from a large randomized trial. Clin Infect Dis. 2012;54(8):1080-1090.

13. Lyden $P$, et al. Phase 1 safety, tolerability and pharmacokinetics of 3K3A-APC in healthy adult volunteers. Curr Pharm Des. 2013;19(42):7479-7485.

14. Mosnier LO, Sinha RK, Burnier L, Bouwens EA, Griffin JH. Biased agonism of protease-activated receptor 1 by activated protein $C$ caused by noncanonical cleavage at Arg 46. Blood. 2012;120(26):5237-5246.

15. Otani A, Takagi H, Oh H, Koyama S, Honda Y. Angiotensin II induces expression of the Tie2 receptor ligand, angiopoietin-2, in bovine retinal endothelial cells. Diabetes. 2001;50(4):867-875.

16. Finigan $\mathrm{JH}$, et al. Activated protein $\mathrm{C}$ mediates novel lung endothelial barrier enhancement: role of sphingosine 1-phosphate receptor transactiva- tion. J Biol Chem. 2005;280(17):17286-17293.

17. Tong Y, et al. Artemisinin and its derivatives can significantly inhibit lung tumorigenesis and tumor metastasis through Wnt $/ \beta$-catenin signaling [published online ahead of print April 22, 2016]. Oncotarget. doi:10.18632/oncotarget.8920.

18. Zuo S, et al. Artesunate protected blood-brain barrier via sphingosine 1 phosphate receptor $1 /$ phosphatidylinositol 3 kinase pathway after subarachnoid hemorrhage in rats [published online ahead of print January 28, 2016]. Mol Neurobiol. doi:10.1007/s12035.016.9732-6.

19. Van Sluis GL, et al. Endogenous activated protein $\mathrm{C}$ limits cancer cell extravasation through sphingosine-1-phosphate receptor 1-mediated vascular endothelial barrier enhancement. Blood. 2009;114(9):1968-1973.

20. Fedson DS, Jacobson JR, Rordam OM, Opal SM. Treating the host response to ebola virus disease with generic statins and angiotensin receptor blockers. MBio. 2015;6(3):e00716. 